\title{
Robustness Evaluation for Harmonic Suppression of LCL-Type Converter Based on Converter-Side Current Feedback Strategy Under Weak and Distorted Grid
}

\author{
Qi WANG, Wenping QIN, Xiaoqing HAN, Peng WANG, Lei WANG, and Ye ZHANG
}

\begin{abstract}
Due to the existence of grid impedance, the grid voltage often contains low order background harmonics, which will deteriorate the current quality of grid connected converters. In view of this situation, considering the delay effect and weak grid, the robustness of two single loop control modes of LCLtype grid connected converter, namely grid-side current feedback (GCF) and converter-side current feedback (CCF), is evaluated to improve the current quality of the grid current under distorted grid. Through the analysis and demonstration of above impedance models, CCF has the advantages of excellent stability and excellent power quality. Then, a direct harmonic suppression strategy based on CCF is proposed. The strategy takes the converter side current which contains richer low harmonic content as harmonic information source, and the control effect is more effective than the traditional strategy which uses capacitor current as harmonic information source. Without additional control strategy and controller, the hardware cost is reduced and algorithm complexity is simplified. The simulation and experimental results verify the correctness and feasibility of the theoretical analysis.
\end{abstract}

Index Terms - Grid current, harmonic suppression, LCL-type converter, robustness, voltage distortion.

\section{INTRODUCTION}

$\mathrm{I}^{\mathrm{N}}$ $\mathrm{N}$ recent years, renewable energy solutions based on the usage of wind power and photovoltaic panels have been widely studied and used in power system. Due to the longdistance distribution of the source and load (SL) in renewable energy power systems, the transmission line impedance and

Manuscript received June 16, 2020; accepted September 14, 2020. Date of publication June 30, 2021; date of current version June 15, 2021. This work was supported in part by the National Key Research and Development Plan under Grant U1610121, in part by Natural Science Foundation of Shanxi Province under Grant 201701D121134, in part by Major Science and Technology Projects of Shanxi Province under Grant 20181102028, and in part by Science and Technology Project of Shanxi Electric Power Company under Grant SGTYHT/18-JS-202. (Corresponding author: Qi Wang.)

Q. Wang is with the College of Electric Power, Civil Engineering and Architecture, Shanxi University, 030013, No.36, Honggou South Street Taiyuan, Shanxi Province, China (e-mail: 343366922@qq.com; 18234083503@163.com).

W. Qin, X. Han, L. Wang, and Y. Zhang are with the College of Electrical Engineering, Taiyuan University of Technology, 030024, China.

P. Wang is with the College of Electrical Engineering, Nanyang Technology University, Singapore, S639798 (e-mail: epwang@ntu.edu.sg).

Digital Object Identifier 10.24295/CPSSTPEA.2021.00015 transformer leakage reactance have to be taken into account. The point of common coupling (PCC) is a common point of weakness in the grid [1]-[2]. This may lead to stability and power quality problems of grid connected converter. Namely, the low-order harmonics generated by a non-linear load leads to the background voltage harmonics at the PCC due to the grid impedance, which further affects the connection between the grid and converter. In addition, the high-order switching harmonics generated by the operation of the equipment connected to the power electronic grid further degrade the grid power quality [3]-[4].

The LCL-type converters have been widely used in renewable energy grid-connected systems due to their excellent performance in high-frequency harmonic attenuation [5]. However, the phenomenon of resonance exists due to the circuit structure of the LCL-type converters. The active damping method can be employed to avoid these problems such as high hardware cost and reduced power transmission efficiency caused by passive damping method [6]-[15]. Besides, the performance of the control system should be studied considering the digital delay, which causes a phase lag in the control system and threatens system stability and antidisturbance ability. For the converter-side current feedback $(\mathrm{CCF})$ and grid-side current feedback (GCF), stability can be achieved without using a damping strategy if specified digital delay requirements are met [8],[16]. Many measures have been proposed by scholars to reduce the delay, including the double sampling method, the impedance reshaping method and the pole-zero delay compensation method [14]-[17]. An improved sampling technique is proposed in [14], which can reduce the delay time by sampling the signal twice in each carrier cycle. By adopting the state observer, the signal of the previous cycle can be used to predict the signal of the next cycle, so as to reduce the sampling delay caused by digital delay. However, this method requires additional computing resources, and the observer is extremely sensitive to the to the small range variation of parameters. For example, the parameter values of resistance and inductance will change with the length of current passing time, which may lead to estimation or prediction error [15]. The delay effect can also be effectively reduced by adding low-pass filter [5] and sampling reconstruction [14]. In addition, there are many researches on 
converter performance and delay compensation methods in weak grid environment. Among them, reference [16] mainly focuses on the influence of weak grid on the inherent resonant frequency of LCL converter, so as to improve the stability of control system. However, the method to reduce the delay is still double sampling method, which is similar to reference [14]. It should be noted that they will increase the complexity of the control algorithm and the burden of experimental calculation.

In terms of power quality, voltage distortion has an important impact on the control performance of LCL converter. A full voltage feedback control strategy is proposed in reference [7], which achieves ideal harmonic suppression effect theoretically. In fact, proportional voltage feedback can not only suppress most of the low order harmonics, but also improve the dynamic response of the control system. However, in the absence of selective harmonic suppression, low order harmonics, that is, below 10th order harmonics, are suppressed, and the attenuation ability decreases with the increase of harmonic order. Repetitive controller is used to suppress the target harmonic. However, the repetitive controller is more sensitive to noise and has infinite gain at Nyquist frequency [11]-[12]. According to the special structure of LCL converter, the capacitor current is used as the harmonic information source in reference [18]. However, the equivalent circuit model shows that there are many low order harmonics and fundamental frequency currents flowing through the LCL structure under the non ideal grid conditions, which is worthy of further exploration and improvement. In the existing research, there is rarely in-depth analysis on the LCL structure multi loop impedance models, and the influence of distorted voltage on the circuit is not discussed. In addition, for the traditional strategy [18], the input state variable of harmonic controller (HC) represents is sum of converter side current and capacitor current. The control effect may be masked by the converter side current. In addition, in the closed-loop control system, the control of capacitor current will reduce the stability of the system [19], [20].

To address all the mentioned problems, in this paper, a direct, simple and effective strategy has been proposed to enhance the robustness of the LCL-type converter when the grid voltage contains background harmonics. By using the proposed strategy, the quality of the grid-into current can be improved without using an additional sensor to sample the capacitor current.

The main contributions of this paper can be summarised as follows:

i. The delay effect and the digital delay reduction methods are considered, and the reasons for selecting CCF as a control method in this paper are expanded and analysed.

ii. The impedance models of two different loops of the LCL structure under the condition of grid distortion, which are referred to as harmonic impedance models in the following sections, are analysed in-depth. The analysis results indicate that in the distorted grid, at lower-order harmonics, the inductor loop provides richer and more accurate information on harmonics than the capacitor loop.

iii. The change in the converter stability with the adoption of

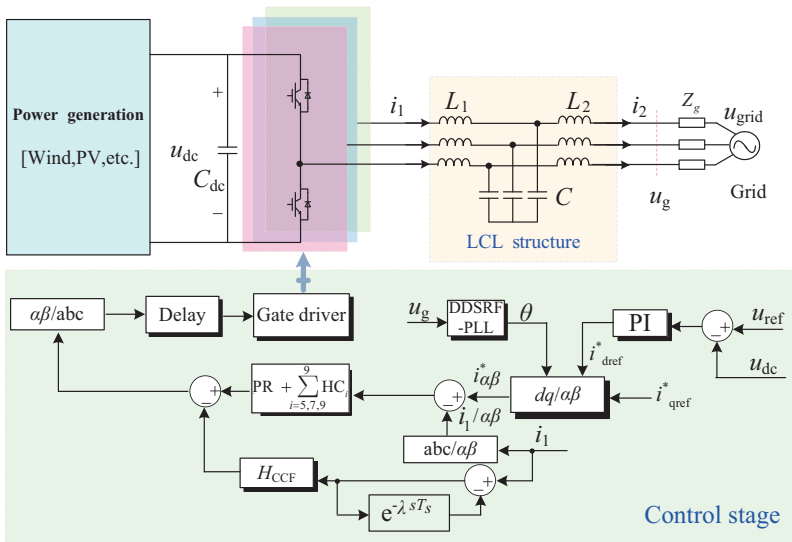

Fig. 1. Structure of the LCL-type grid-tied converter.

TABLE I

Parameters of the Main and Control Circuits

\begin{tabular}{lc||lc}
\hline \hline Parameter & Value & Parameter & Value \\
\hline$U_{\mathrm{g}}$ & $80 \mathrm{~V}$ & $K_{\mathrm{p}}$ & 0.12 \\
$L_{1}$ & $1.5 \mathrm{mH}$ & $K_{\mathrm{i}}$ & 53 \\
$L_{2}$ & $1.5 \mathrm{mH}$ & $k_{\mathrm{HC}}$ & 300 \\
$C$ & $10 \mu \mathrm{F}$ & $w_{\mathrm{c}}$ & 25 \\
$U_{\mathrm{dc}}$ & $200 \mathrm{~V}$ & $L_{\mathrm{g}}$ & $3 \mathrm{mH}$ \\
\hline \hline
\end{tabular}

the capacitor current is investigated, and a simple and effective strategy is proposed to suppress the low-order harmonics caused by grid distortion.

The remaining of the paper is organised as follows: In Section II, a mathematical model of the LCL-type converter with a digital delay is introduced. The impedance models under the distorted grid condition are analysed in detail in Section III. In Section IV, the harmonic suppression mechanism and the stability of a system using the traditional control strategy and the proposed control strategy are investigated, and an effective strategy is proposed. In Section V, the simulation and experimental results verify the proposed strategy. Finally, the main conclusions are drawn in Section VI.

\section{Model of LCL-Type Converter With Digital Delay EFFECt}

The structure of the LCL-type grid-tied converter is shown in Fig. 1, and the special parameters of the main and control circuit are given in Table I.

In Fig. $1, i_{1}, i_{2}$ and $C$ denote the converter-side current, the grid-side current and the LCL structure capacitor, respectively, $C_{\mathrm{dc}}$ denotes the DC side capacitor, and $Z_{\mathrm{g}}$ denotes the impedance of the grid, which represents the weakness of the grid. The delay link represents the overall time delay including the delays caused by analog-to-digital conversion, controller computation, duty-ratio update and pulse with modulation (PWM) delay. The control structure mainly analyzed in this paper is Fig. 1. Furthermore, the related description and 


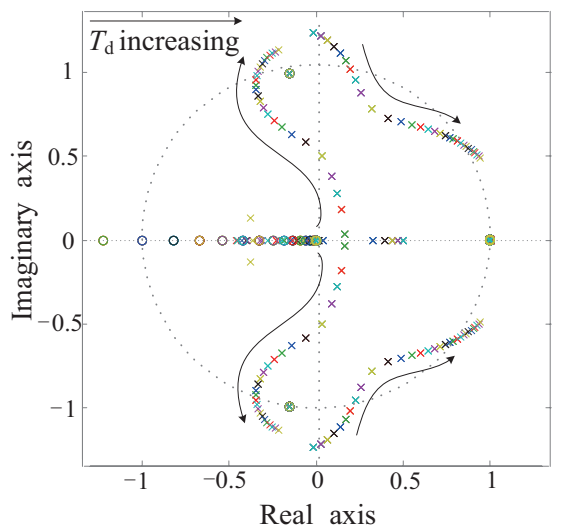

(a)

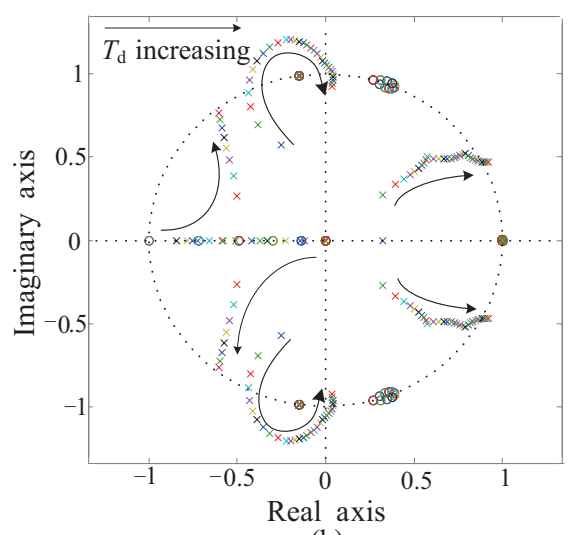

(b)

Fig. 2. Zero-pole figures of (a) GCF and (b) CCF.

improvement of the control structure in Fig. 1 will be described later.

The zero-pole figures of the closed-loop trans-function of the GCF and CCF are displayed in Fig. 2, where it is shown that the performances of the GCF and CCF are inversely affected by the delay. In the theoretical analysis and simulation, the Pade function and Taylor formula have been used to approximately equivalent $\exp \left(-s T_{\mathrm{d}}\right)$ in $s$ domain.

Unlike the CCF, in the case of the GCF, as $T_{\mathrm{d}}$ increases, the system transitions from stable to unstable. In other words, an appropriate delay value is beneficial to the GCF stability, although this is in conflict with the original goal of reducing the delay to enhance converter robustness. In addition, increasing the delay can lead to the phase lag, which potentially threatens system stability. The stable frequency interval of the GCF is $f_{\text {res }} \in\left(f_{\mathrm{s}} / 6, f_{\mathrm{s}} / 2\right)$, where, $f_{\text {res }}$ represents the resonance frequency, and $f_{\mathrm{s}}$ represents the sampling frequency [21], [22].

Generally, as shown in the blue line in Fig. 3, $f_{\text {res }}$ decreases with the grid impedance $Z_{\mathrm{g}}$ increases. The resonance frequency is likely to reduce below the critical value due to variations in grid impedance under weak grid conditions. The larger the impedance of the power grid is, the weaker the power grid is and the smaller the resonance frequency is. The larger value of the impedance is, the weaker grid is and the smaller the resonance frequency is. When the critical frequency remains unchanged, it is easy for GCF to cross the critical frequency.

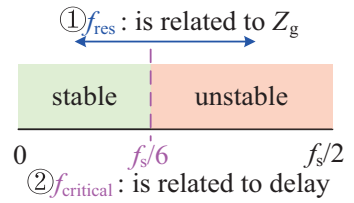

(a)

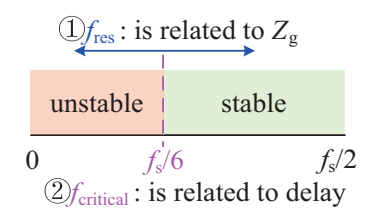

(b)

Fig. 3. Stable regions of (a) CCF and (b) GCF.

At this time, the system changes from stable to unstable, and the greater the impedance of the power grid, the greater the risk of instability. For CCF, the increase of grid impedance is beneficial to system stability.

Furthermore, as shown in the purple line in Fig. 3, with the reduction in the delay, the critical frequency $f_{\mathrm{s}} / 6$ will increase, thus narrowing the stable interval. Specifically, the larger the phase lag caused by the delay effect is, the smaller the critical frequency is. For GCF When the grid impedance remains constant, the larger the delay, the smaller the critical frequency, and the larger the stability region. For CCF, the larger the delay, the smaller the stability region of the system. In this paper, the CCF is adopted to avoid the disadvantages. For instance, the GCF is not conducive to the implementation of the delay reduction strategy, it is unable to directly carry out the current limiting protection of the converter, and its stability is poor. The stable interval of CCF is $f_{\text {res }} \in\left(0, f_{\mathrm{s}} / 6\right)$.

At the same time, current protection also requires a converter-side current. In [23], the authors proposed a simple and effective unit delay feedback strategy to mitigate the negative effects of delays, the method is adopted in this paper to alleviate the potential instability caused by time delay and grid impedance. The unit delay feedback strategy is shown in Fig. 3. Here is a brief description. The phase near the resonance frequency decreases due to the delay. According to the theory of unit negative feedback in automatic control theory, the most direct way is to connect a lead-lag link in the equivalent delay link of the system in view of the delay effect. The design goal of this link needs to achieve the following effect: The phase at the resonant frequency should be raised. At the same time, the low frequency gain can be guaranteed when the phase lag caused by the delay link is relieved. Considering the above factors, [23] constructs a unit delay feedback link, which is the strategy used in this paper and it will not be discussed in detail in this paper. In Fig. 4, which is a simplified graph of Fig. 1, $G_{\mathrm{d}}$ denotes the overall delay of the control system, $G_{i}$ denotes the current controller, which is a PR \& HC controller, and $\lambda$ represents the coefficient of the unit delay feedback strategy. The one-order high power filter $H_{\mathrm{CCF}}$ could be adopted when necessary. 


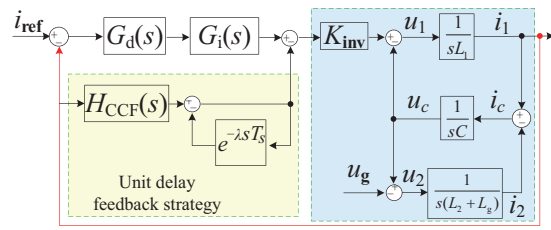

Fig. 4. Block diagram of the CCF.

Based on Fig. 4, the following equations can be obtained.

$$
\begin{gathered}
G_{\mathrm{d}}(s)=e^{-1.5 s T_{\mathrm{s}}}=e^{-s T_{\mathrm{d}}} \\
i_{1}=\frac{G_{i}(s) G_{1}(s) e^{-s T_{\mathrm{d}}}}{1+G_{i}(s) G_{1}(s) e^{-s T_{\mathrm{d}}}} i^{*}-\frac{G_{1}(s) G_{2}(s)}{1+G_{i}(s) G_{2}(s) e^{-s T_{\mathrm{d}}}} u_{\mathrm{g}} \\
i_{2}=\frac{G_{i}(s) G_{1}(s) G_{2}(s) e^{-s T_{\mathrm{d}}}}{1+G_{i}(s) G_{1}(s) e^{-s T_{\mathrm{d}}}} i^{*} \\
-\frac{G_{1}(s) G_{2}(s) G_{2}(s)+s C G_{i}(s) G_{1}(s) G_{2}(s) e^{-s T_{\mathrm{d}}}+s C G_{2}(s)}{1+G_{i}(s) G_{1}(s) e^{-s T_{\mathrm{d}}}} u_{\mathrm{g}}
\end{gathered}
$$

where,

$$
\begin{gathered}
G_{1}(s)=\frac{s^{2} L_{2} C+1}{s^{2} L_{1} L_{2} C+s\left(L_{1}+L_{2}\right)}, \\
G_{2}(s)=\frac{1}{s^{2} L_{2} C+1}, \\
G_{i}(s)=K_{\mathrm{p}}+\sum_{\substack{i=2 n+1 \\
n=0,1,2, \ldots}}^{\infty} \frac{K_{\mathrm{HCi} i} s}{s^{2}+w_{\mathrm{c}} s+w_{\mathrm{i}}^{2}} .
\end{gathered}
$$

In (1)-(6), $G_{i}(s)$ represents the PR\&HC controller that consists of the PR and HC sub-controllers; the PR represents the proportional-resonance controller, where $K_{\mathrm{p}}$ denotes the proportional coefficient, $w_{\mathrm{c}}$ denotes the bandwidth coefficient, and $w_{0}$ denotes the fundamental angular frequency; the $\mathrm{HC}$ represents the harmonic controller that is connected in parallel with the PR, and where $K_{\mathrm{HC}}$ denotes the gain and $w_{\mathrm{i}}$ denotes the harmonic-order angular frequency.

\section{IMPEDANCE MODEL OF LCL-TyPe CONVERTER IN DISTORTED GRID}

Similar to a person with weak health who is prone to illness, a weak grid is highly susceptible to the background harmonic voltages, which can lead to a low-quality grid current. Based on (3), the impedance model under the condition of grid distortion or grid harmonics can be expressed as:

$$
Z_{\mathrm{g}}=\frac{u_{\mathrm{g}}}{i_{2}}=\frac{1+G_{i}(s) G_{1}(s) e^{-s T_{\mathrm{d}}}}{G_{1}(s) G_{2}(s) G_{2}(s)+s C G_{i}(s) G_{1}(s) G_{2}(s) e^{-s T_{\mathrm{d}}}+s C G_{2}(s)}(7)
$$

The equivalent circuit model of grid connected converter can be obtained, and it is shown in Fig. 5. As presented in Fig. 5,

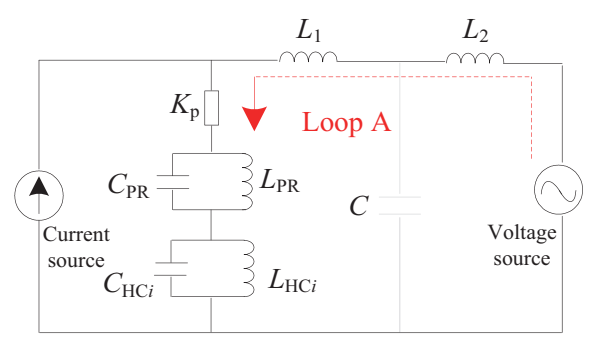

(a) Loop A

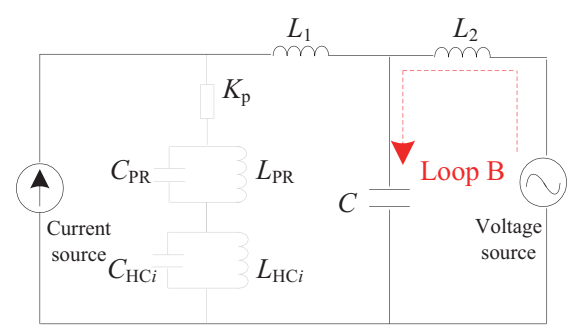

(b) Loop B

Fig. 5. Circuit schemes of two loops of a distorted grid.

there are two loops for the voltage harmonics, loop A and loop $\mathrm{B}$, which can be presented in the form of harmonic currents. When the voltage at PCC point is distorted, the harmonic voltage will flow through two loops in the form of harmonic current through the output impedance of the converter. At this time, the impedance characteristics of each loop determine the content or proportion of harmonic current in the loop. Also, in Fig. $5, K_{\mathrm{p}}$ denotes the proportion gain of the PR controller; $C_{\mathrm{PR}}$ and $L_{\mathrm{PR}}$ denote the capacitor and inductor respectively in the equivalent circuit model of the PR controller; $C_{\mathrm{HC} i}$ and $L_{\mathrm{HC} i}$ respectively denote the capacitor and inductor in equivalent circuit model of $\mathrm{HC}$ controller, where $i$ denotes the $i$ th order harmonic.

Considering the controller, the impedance frequency domain analysis of the two loops is shown in Fig. 6. When the harmonic frequency is lower than $750 \mathrm{~Hz}$, the amplitude gain or admittance of loop $\mathrm{A}$ is higher than that of loop $\mathrm{B}$, and the harmonic current mainly flows through loop A. The traditional control strategy of LCL converter uses the capacitor current as harmonic source whose frequency domain characteristic is loop B [16], [18], [22]. However, loop B presents high impedance characteristics in the low frequency domain, and only the frequency harmonic currents above $750 \mathrm{~Hz}$ are allowed to pass through. In practice, the voltage background harmonics are mostly within the 15 th harmonic [2], [14]. According to Fig. 6, most of the harmonic current flows to loop A and a small part of that to loop B. The harmonic information in loop $\mathrm{A}$ is more abundant and accurate. When control system samples the loop A current, the harmonic information can be obtained more accurately, and the harmonic in grid current could be suppressed more effectively.

Fig. 7 shows the path diagram of all order harmonic currents according to above mentioned. Loop F denotes the current channel in fundamental frequency region, Loop $\mathrm{R}$ denotes the current channel in resonance frequency region, and Loop $\mathrm{H}$ 

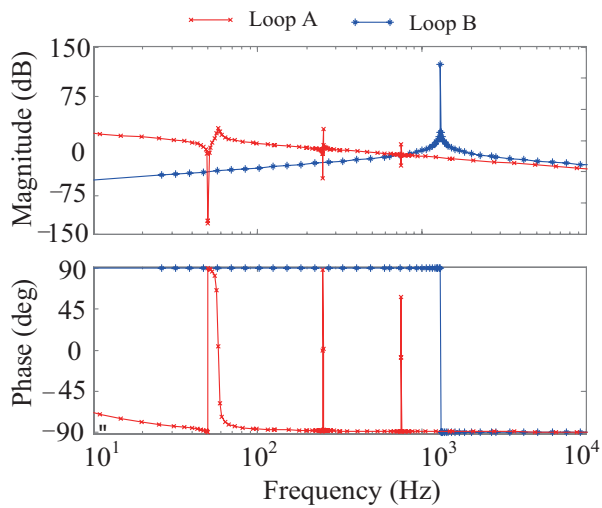

Fig. 6. Impedance of the two loops

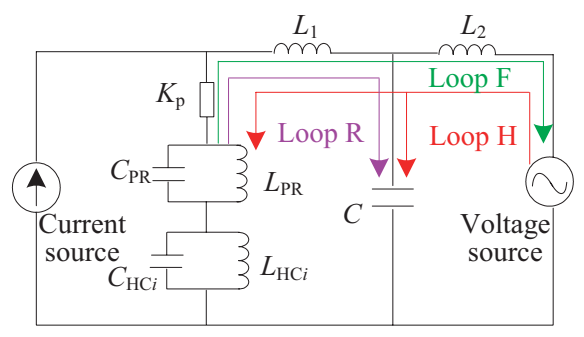

Fig. 7. Path diagram of the all order harmonic currents.

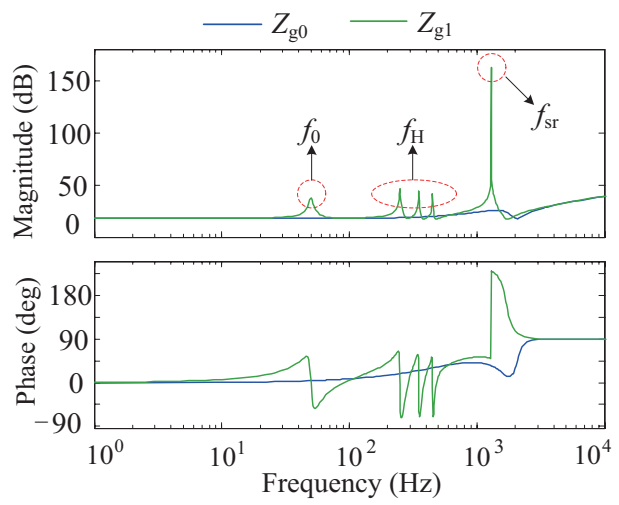

Fig. 8. Comparison of $Z_{\mathrm{g} 0}$ and $Z_{\mathrm{g} 1}$.

denotes the harmonic frequency current channel caused by the background harmonics of voltage.

The frequency domain characteristics of the circuit model determine the main flow direction of the harmonic current, the above analysis is the theoretical basis of the strategy proposed in the next section.

\section{Proposed Grid-Current Quality Enhanced Strategy For CCF CONTROL System}

\section{A. Harmonic Suppression Mechanism and Stability Analysis}

In this paper, an $\mathrm{HC}$ is employed as controller to suppress grid current with low-order harmonics caused by distorted voltage at PCC. Fig. 8 shows bode of traditional harmonic suppression strategy. That is, the harmonic source comes from the capacitor current of loop B. In Fig. 8, $Z_{\mathrm{g} 1}$ denotes the traditional strategy with the PR \& $\mathrm{HC}$ controller, which are composed of $\mathrm{HC}_{5}$, $\mathrm{HC}_{7}$ and $\mathrm{HC}_{9}$. Then, $Z_{\mathrm{g} 0}$ denotes the harmonic suppression strategy with a PR controller, which uses the $i_{1}$ in loop A as the information source of harmonic for controlled.

In additional, series resonance is stimulated by $Z_{\mathrm{gl}}, C$ and $L_{2}$ in loop B in Fig. 8. This may lead to the unstable operation and even weaken the control system robustness. Furthermore, $f_{0}$ represents the fundamental frequency, $f_{\mathrm{H}}$ represents the harmonic frequency of the $\mathrm{HCs}$, which, in this paper, is composed of $\mathrm{HC}_{5}, \mathrm{HC}_{7}$ and $\mathrm{HC}_{9}$, and $f_{\mathrm{sr}}$ represents the series resonance frequency of loop $\mathrm{B}$, where:

$$
f_{\mathrm{sr}}=\frac{1}{2 \pi} \sqrt{\frac{1}{C L_{2}}} .
$$

When the traditional strategy is adopted, the introduction of $i_{\mathrm{c}}$ into the control loop can bring hidden dangers to system stability, Fig. 9 shows the related control structure. Based on the structure of Fig. 1, an independent analysis of branches of $i_{\mathrm{c}}$ is conducted in this section. In Fig. 9, the symbol $w$ represents the resonance angle frequency, and its expression is shown in (9). In addition, the loop formed by red dotted lines represents the signal loop when the capacitor current is used as a harmonic information source, and its root locus is shown in Fig. 10.

$$
w=2 \pi f_{\mathrm{res}}=\sqrt{\frac{L_{1}+L_{2}}{L_{1} L_{2} C}} .
$$

In Fig. 10, obviously, the root locus of the control system is always outside the unit circle when the delay effect is considered, which may lead to the system unstable. Therefore, the capacitor current maybe an unstable factor in control system, and it is not a perfect method to mitigate the negative effects caused by distorted voltage.

\section{B. Proposed Grid Current Enhanced Quality Strategy}

As shown the control structure in Fig. 9, before the controlled signal enters the controller, the input signal of $\mathrm{HC}$ is the sum of converter-side current $i_{1}$ and capacitor current $i_{\mathrm{c}}$. Based on the analysis in Section II, it could be noted that the only a small amount of low order harmonics carried by $i_{\mathrm{c}}$ and it may lead to instability. In this paper, the $i_{1}$ is to be adopted as the harmonic current information source. The bode figures of the related open-loop system are shown in Fig. 11, where the $Z_{\mathrm{g} 2}$ denotes the converter output impedance with the proposed strategy. For comparison, the curve $Z_{\mathrm{g} 1}$ in Fig. 8, which is represented by the green line, is also redraw in Fig. 11.

Obviously, it can be seen from Fig. 11 that the series resonance frequency caused by loop B has been suppressed, and the proposed strategy ensures a high gain in the fundamental frequency region. The phase frequency curve passes through the zero axis once in the positive and negative directions, the Nyquist stability criterion is satisfied. In addition, by adjusting the gain and bandwidth coefficients, as well as by adding HCs, the same effect of low order harmonic 


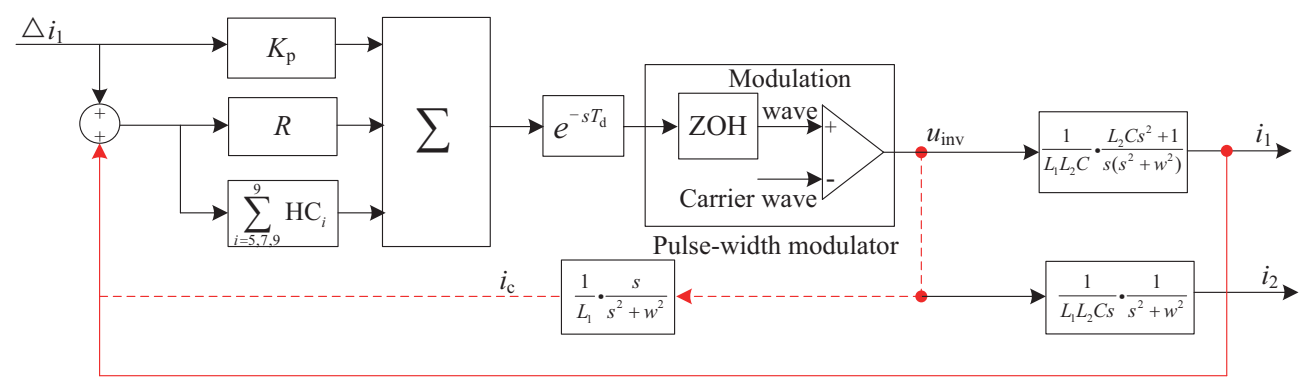

Fig. 9. The control block with traditional and proposed harmonic suppression strategy.

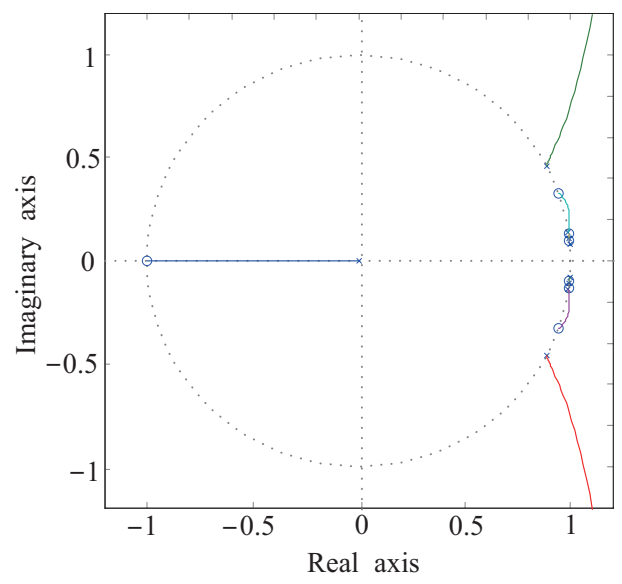

(a) Overall map of root locus

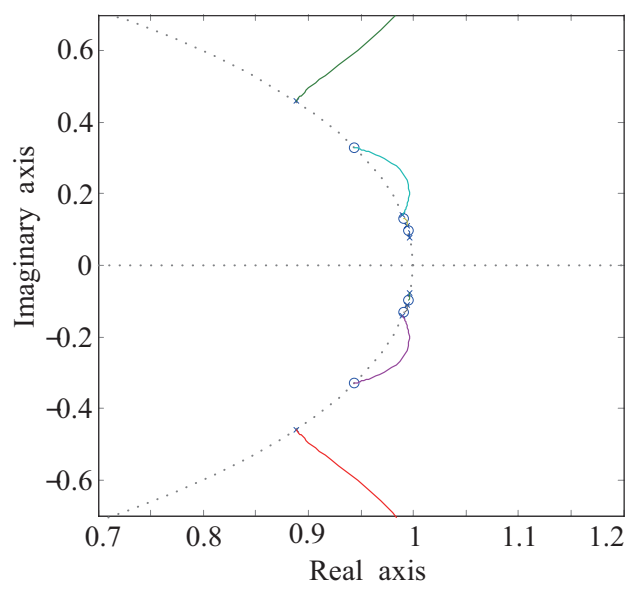

(b) Partial enlargement map of root locus

Fig. 10. Root locus of the capacitor current channel.

current suppression could be realized either.

The loop formed by red solid lines in Fig. 8 represents the signal loop when the converter-side current is used as a harmonic information source. Fig. 12 shows the root locus with the proposed strategy. Compared with the traditional strategy, the proposed strategy does not need sample $i_{\mathrm{c}}$ to form a closedloop control loop. On the premise of ensuring sufficient lowfrequency gain, the root locus falls within the unit circle. Simultaneously, the method reduces the sensitivity to system parameters. When the control parameters are adjusted in a

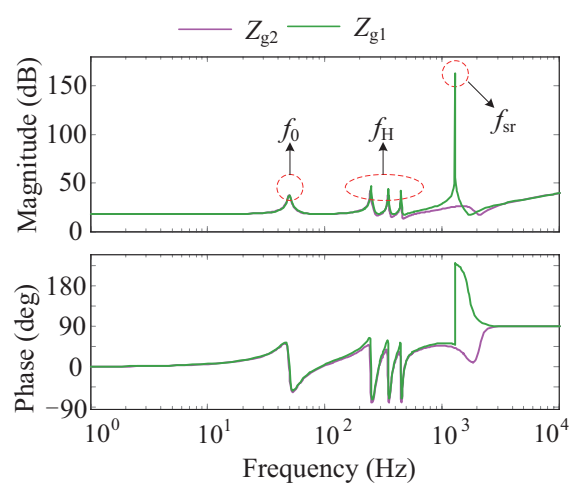

Fig. 11. Comparison of $Z_{\mathrm{g} 2}$ and $Z_{\mathrm{g} 1}$.

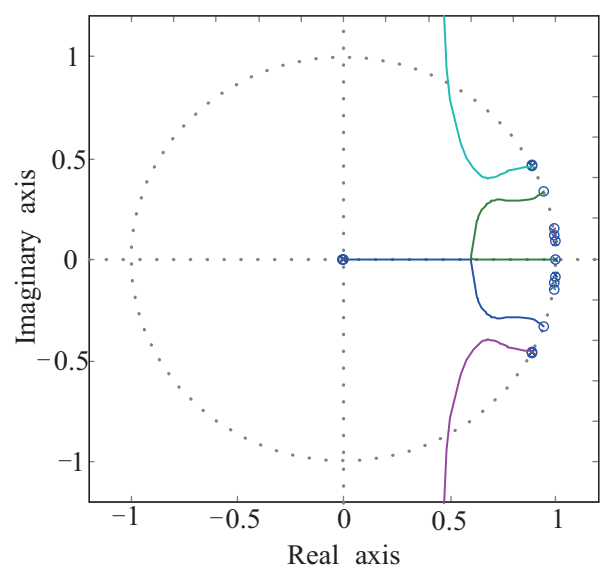

Fig. 12. Root locus of the proposed strategy.

wide range, the system is still stable. So the strategy effectively optimizes the control performance of the grid-tied converter, and improves the stability of the control system.

\section{CASe Studies}

\section{A. Simulation Results}

The simulation model was established using PLECS and Matlab/Simulink to verify the previous analysis. The related parameters are listed in Table I.

In order to verify the effectiveness of the proposed strategy, the $30 \%$ of the 7 th order harmonic is injected into the ideal 

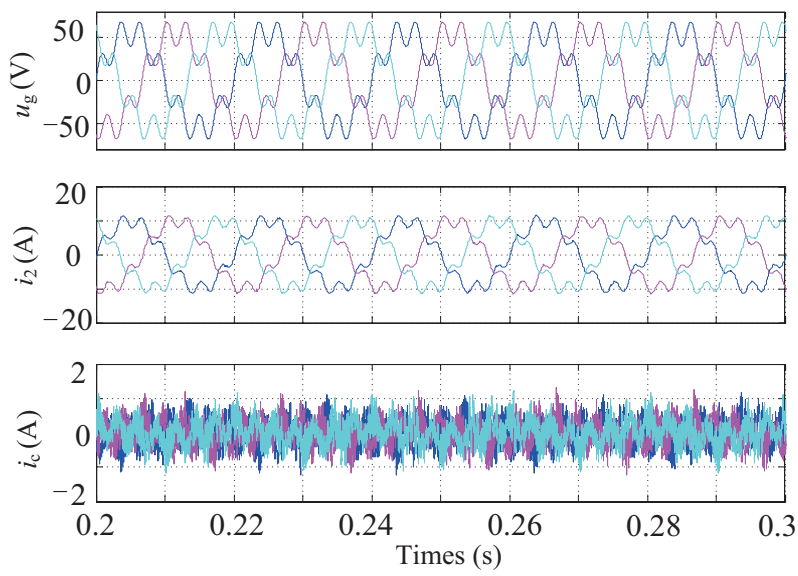

Fig. 13. Voltage and current at the PCC point and capacitor current without any control strategy.

grid voltage to simulate a distorted and weak grid within background harmonics. Fig. 13 shows the grid voltage $U_{\mathrm{g}}$, the grid current $i_{2}$ and the capacitor current $i_{\mathrm{c}}$ under distorted grid conditions without any control strategy. Obviously, the background voltage harmonics significantly worsened the quality of the grid current.

Furthermore, according to Fig. 13, the results of FFT analysis for $i_{2}$ and $i_{\mathrm{c}}$ are shown in Fig. 14. Fig. 14 (a) shows the FFT analysis results of $i_{2}$. Except for the fundamental frequency current, the 7 th order harmonic content is the highest, about $2 \mathrm{~A}$. The resonance frequency is $1.84 \mathrm{kHz}$. At this time, the THD of $i_{2}$ is $21.5 \%$, which is seriously affected by the distorted grid. Fig. 14 (b) shows the FFT analysis results of $i_{\mathrm{c}}$. The resonance current content in $i_{\mathrm{c}}$ is the highest, while the 7th order harmonic content is relatively small, about $0.3 \mathrm{~A}$. The content of low frequency harmonics in $i_{\mathrm{c}}$ is much less than that in $i_{2}$. Which is consistent with the above theoretical analysis.

When the traditional strategy is adopted, that is, the capacitor current $i_{\mathrm{c}}$ in loop $\mathrm{B}$ is used as the harmonic information source for harmonic suppression. The waveforms of grid current and voltage at PCC are shown in Fig. 15. It is not difficult to see that compared with Fig. 13, when the traditional strategy is adopted, the harmonics of grid current is effectively suppressed and the waveform quality is improved. However, the overall THD of current waveform is still large, which indicates that the control effect of traditional strategy is limited. In addition, there is still a risk of periodic instability, and the control system needs further optimisation.

When the proposed strategy is adopted, that is, $i_{1}$ in loop A is sampled as harmonic information source for harmonic suppression. The waveforms of grid current and voltage at PCC are shown in Fig. 16. Compared with Fig. 15, when the proposed strategy is adopted, the harmonic of grid current is effectively suppressed and the waveform becomes highly sinusoidal. The overall distortion rate of the current waveform is small, which shows that the proposed strategy improves the limitation of the traditional control strategy. Without increasing the complexity of the controller, the harmonic

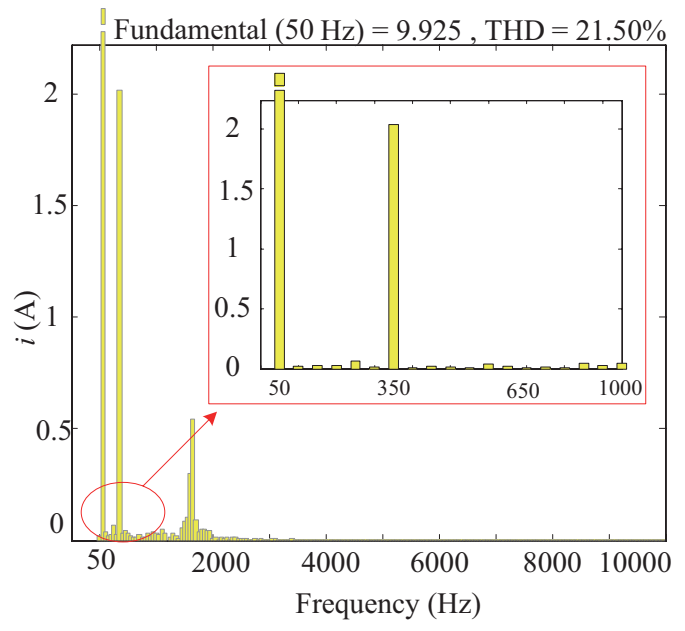

(a) The FFT results of the grid-into current

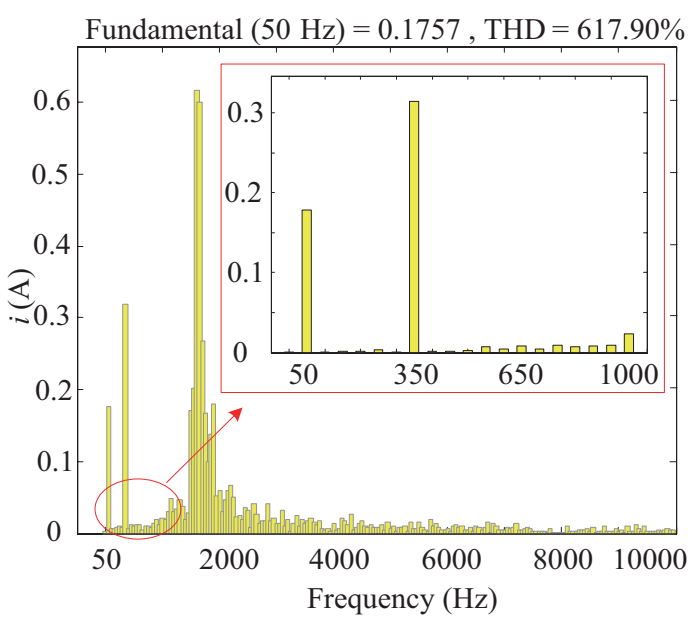

Fig. 14. The FFT results of the grid current and the capacitor current in the distorted grid without any control strategy.
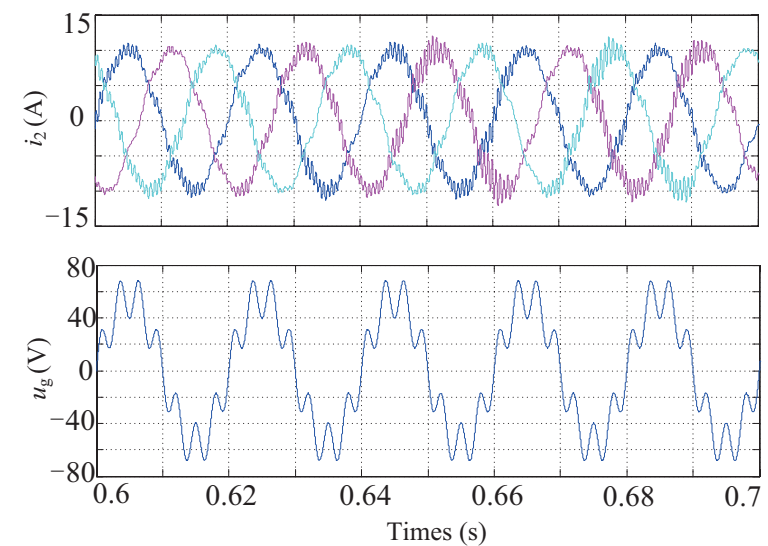

Fig. 15. The waveform of the grid current and PCC voltage of the distorted grid when the traditional strategy is used.

suppression effect is realized more effectively. Furthermore, the convergence has been improved and stability of the current waveform has also been enhanced.

To further illustrate the effectiveness of the proposed strategy, 

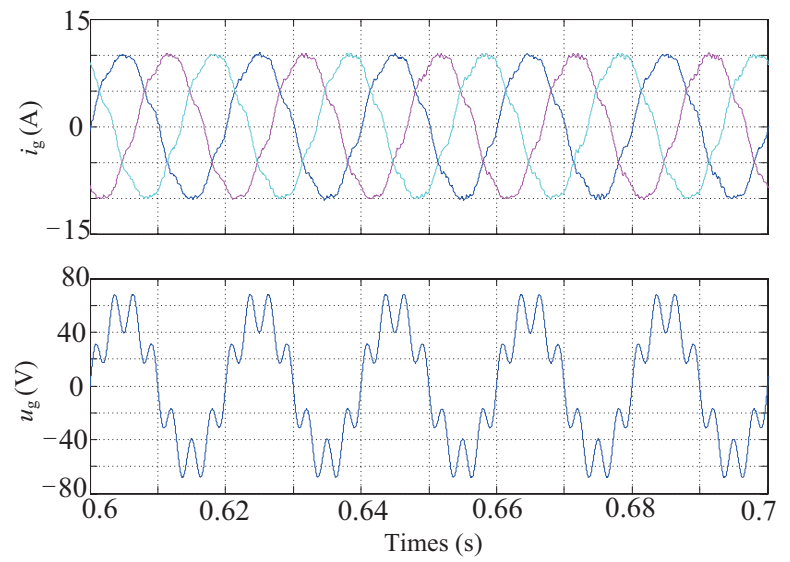

Fig. 16. The waveform of the grid current and PCC voltage of the distorted grid when the proposed strategy is used.

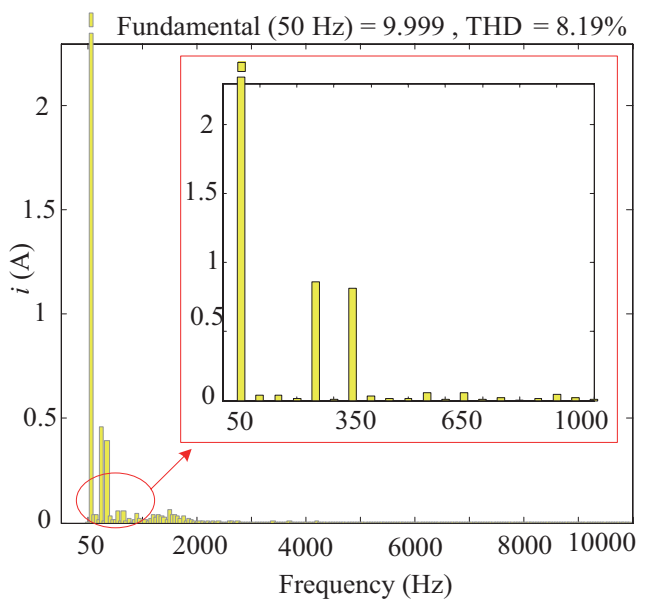

(a) The FFT results of Fig. 15

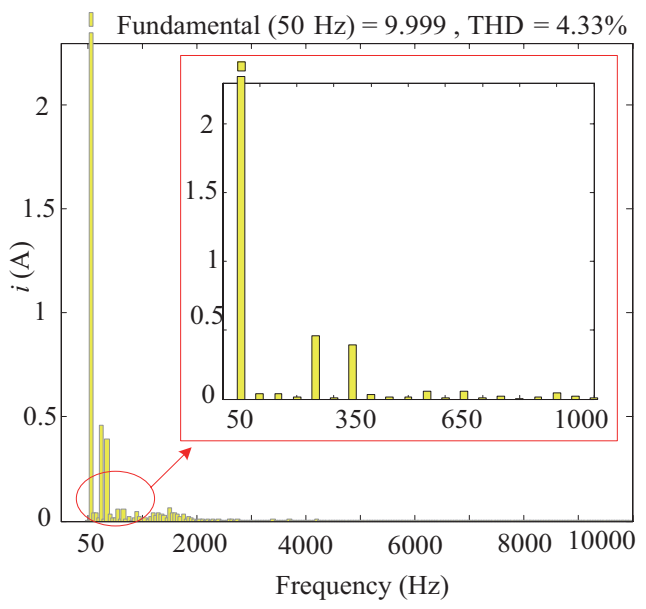

(b) The FFT results of Fig. 16

Fig. 17. The FFT results of the grid current of the distorted grid obtained by using the traditional and proposed strategies.

Fig. 17 shows the FFT analysis results of the current waveform in Figs. 15 and 16. At this time, compared with Fig. 14 (a), THD of grid current is significantly reduced due to the effective suppression of 7th order harmonic as shown in Fig. 17(a).

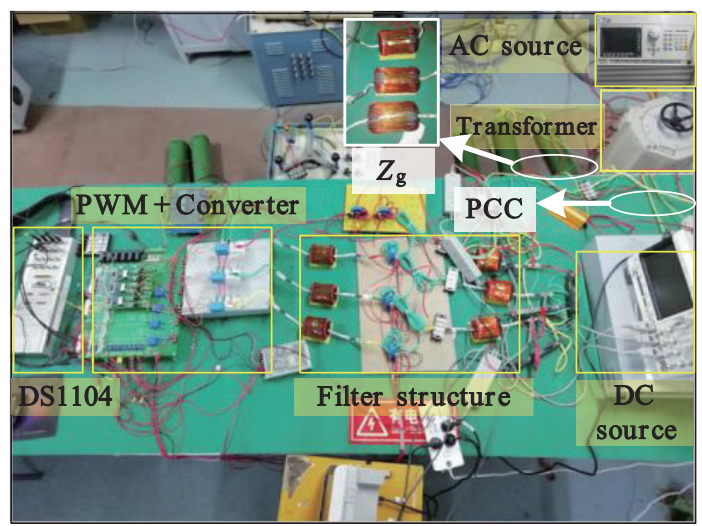

Fig. 18. Hardware structure of the experimental platform.

However, according to the above analysis, it is difficult to make THD meet the requirements of grid connection standard due to the limited suppression effect of traditional strategy. By comparison, Fig. 17(b) shows the FFT analysis results of grid current with the proposed strategy. On the basis of Fig. 17(a), the lower order harmonics are further suppressed and the overall THD of the current is further reduced. It shows that the controller has more targeted and better effect on harmonic suppression by using state variables with more abundant harmonic content in loop A.

\section{B. Experimental Results}

The experimental setup is shown in Fig. 18. In the experiments, the control strategy and algorithm were realised using the dSPACE DS1104 platform, and the non-ideal grid and DC source were implemented using Chroma 61511 and Chroma 62150H-1000, respectively. The LEM LV28-P and LEM LT208-S7/SP1 were used to sample the state variables such as voltage and current at PCC. A K75T60 series insulated gate bipolar transistor (IGBT) produced by Infineon, was used as a switching link in the experimental platform. The experimental parameters are given in Table I.

Here are the experimental results. Fig. 19 shows the current waveform and voltage waveform at PCC point under the ideal grid condition. The pink curve is the result of FFT analysis of phase $A$ current. It can be seen that even in the ideal grid condition, there are still a lot of low order harmonics in the current waveform, among which the 7th order harmonic content is high most.

Fig. 20 shows the waveforms of grid current and voltage when the grid voltage contains the 7 th order harmonic. Fig. 21 shows the corresponding capacitor current waveform. In the above two figures, the working condition at this time is consistent with that in Fig. 13. In Fig. 20, the grid current is seriously distorted by the distorted grid voltage, which contains a large number of voltage background harmonics. According to the FFT results, the 7th order harmonic is the main reason for its distortion. In Fig. 21, the capacitor current content is small, and the 7th order harmonic contained in it is approximately 


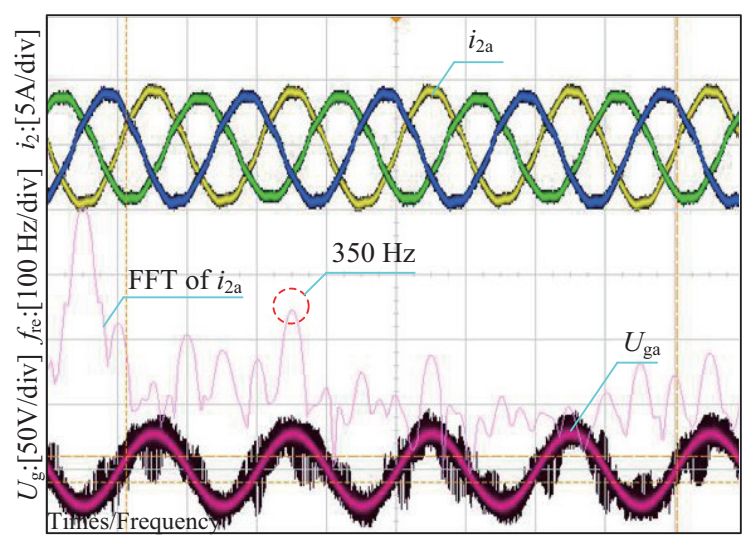

Fig. 19. Grid current and voltage of the ideal grid.

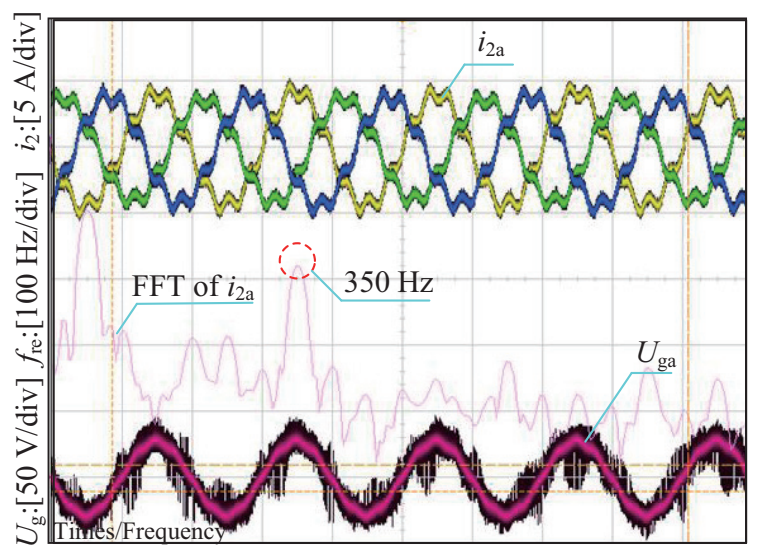

Fig. 20. Grid current and voltage of the distorted grid.

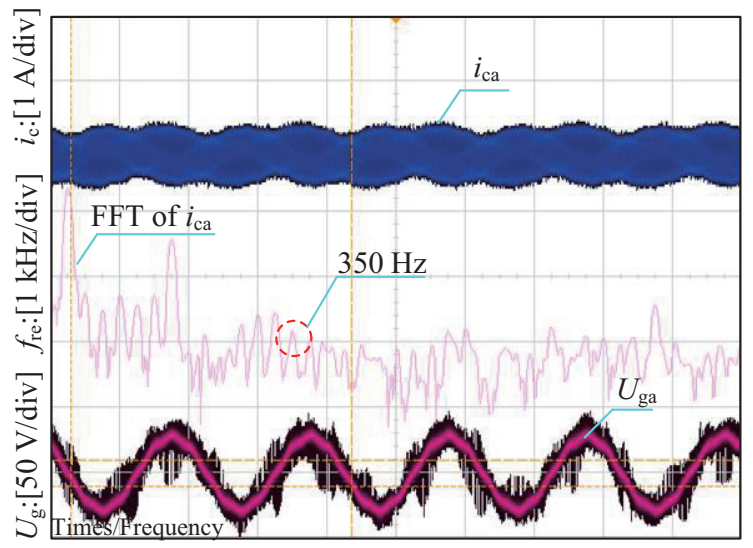

Fig. 21. Capacitor current and voltage of the distorted grid.

zero. It shows that the background harmonics of the grid voltage mainly flow through the inductor loop in the form of low-frequency harmonic current, and the capacitor current mainly provides the path for the high-frequency current. The low frequency harmonic content of the former is higher than that of the latter.

Figs. 22 and 23 show the waveforms of grid current and voltage at PCC after adopting the traditional strategy and

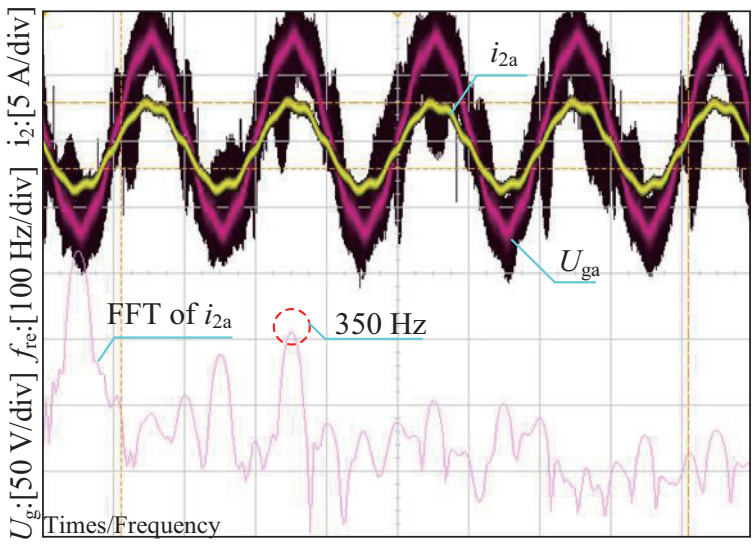

Fig. 22. Single phase of the grid current and voltage when the traditional strategy is adopted.

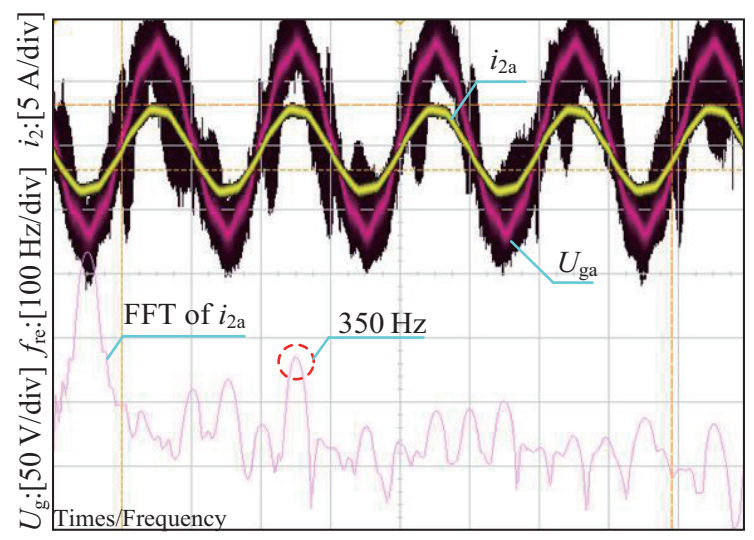

Fig. 23. Single phase of the grid current and voltage when the proposed strategy is adopted.

the proposed strategy respectively. It can be seen that in Fig. 22 , the traditional strategy can effectively suppress the 7 th order harmonic of grid current and restore its waveform to sinusoidal. However, due to the limited low order harmonic content of the harmonic information source, it is difficult for the controller to effectively control the low order harmonic. In addition, the control system also has the risk of instability. In Fig. 23, after using the proposed strategy, the 7th order harmonic can be suppressed to the greatest extent, the current waveform is highly sinusoidal, and the grid current waveform is equivalent to that under the ideal grid condition. It shows that the proposed strategy shows the enhanced robustness even when the grid voltage contains background harmonics.

Furthermore, in order to verify the dynamic characteristics of the proposed strategy before and after enabling. Fig. 24 shows the experimental results of $i_{1}, i_{2}$ and grid voltage. When the proposed strategy is enabled, the current quality of loop A is significantly improved and the harmonics are effectively suppressed. Similarly, Fig. 25 shows the experimental results of three-phase current. It can be seen that after the proposed strategy is enabled, the current waveform has almost no impact and the transient characteristics are good. This shows that the proposed strategy has better transient stability. 


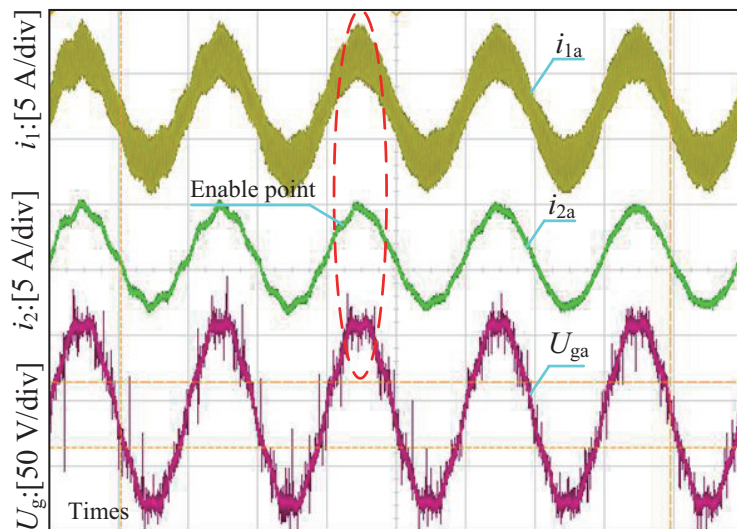

Fig. 24. Single phase of the grid current and voltage when the proposed strategy is adopted.

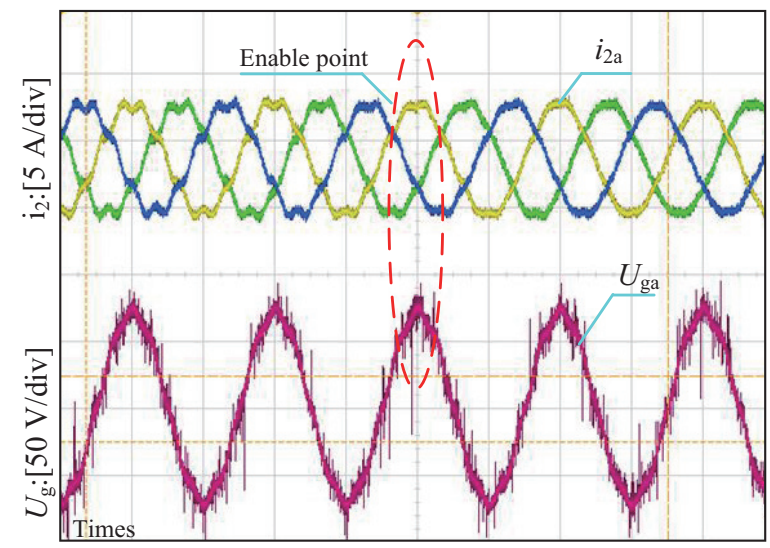

Fig. 25. Grid current and PCC voltage when the proposed strategy is adopted.

TABLE II

Content of the Seventh-Order Harmonic and the THD of the Grid Current

\begin{tabular}{ccccc}
\hline \hline Case & 1 & 2 & 3 & 4 \\
\hline Simulation & $0.012 / 1.4$ & $0.21 / 21.5$ & $0.07 / 8.19$ & $0.035 / 4.33$ \\
Experimental & $0.03 / 3.1$ & $0.29 / 31$ & $0.08 / 9.2$ & $0.042 / 4.8$ \\
\hline \hline
\end{tabular}

P.u.:the seventh-order harmonic/THD (\%).

\section{Result Analysis}

Table II shows the comparison of the unit value (p.u.) of the seventh-order harmonic current in the grid current and the THD of the grid current based on the experimental and simulation results.

Cases 1-4 represent the ideal voltage conditions, the distorted grid conditions without any control strategy, the distorted grid conditions with the traditional control strategy and the distorted grid conditions with the proposed control strategy, respectively.

Furthermore, according to Table II, combined with the simulation and experimental results, Fig. 26 shows the line diagram of the 7th order harmonic content and THD of grid current under various working conditions. It can be seen that

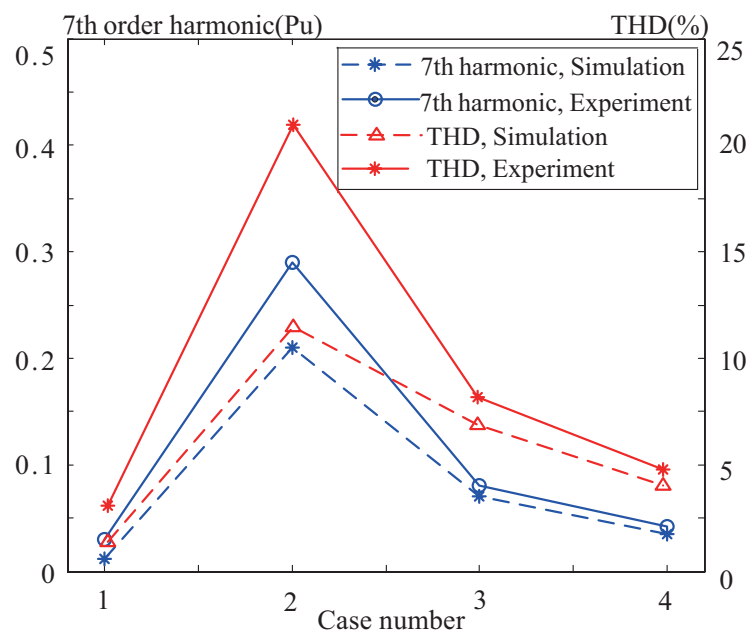

Fig. 26. Comparison of the simulation and experimental results.

trend of the curve of experimental results is consistent with the simulation results. Due to the influence of uncertain factors such as environment, the control effect of experiment is difficult to achieve the same ideal effect as simulation. Convincingly, the related performance indexes have been optimized.

\section{CONCLUSION}

In this paper, the impedance model of different loops in the LCL-type converter is introduced and analyzed in detail to find a simpler and more effective method to improve the quality of the grid current under the distorted grid condition. Firstly, the stability of the two control modes of GCF and CCF is elaborated, and the superiority of CCF is demonstrated. In addition, for the traditional harmonic suppression method of CCF, a theoretical analysis is carried out, and the limitation of using traditional capacitor current as a harmonic information source is analyzed. Furthermore, a direct harmonic current control method is proposed as a supplement to the traditional control method. Compared with traditional control methods, this strategy uses fewer sensors and greatly reduces hardware costs. Due to the simple structure and accurate harmonic information, its performance is better than traditional strategy in terms of grid current quality and system stability. The experiment proves the advantages and effectiveness of the proposed strategy. Related results show that this strategy has better control effect than traditional control strategy.

\section{REFERENCES}

[1] J. Xu, S. Xie, and T. Tang, "Active damping-based control for gridconnected LCL-filtered inverter with injected grid current feedback only," in IEEE Transactions on Industrial Electronics, vol. 61, no. 9, pp. 4746-4758, Sep. 2014.

[2] C. C. Gomes, A. F.Cupertino, and H. A. Pereira, "Damping techniques for grid-connected voltage source converters based on LCL filter: An overview," in Renewable \& Sustainable Energy Reviews, vol. 81, no. 1, pp. 116-135, 2018.

[3] D. Yang, X. Ruan, and H. Wu, "Impedance shaping of the grid-connected inverter with LCL filter to improve its adaptability to the weak grid 
condition," in IEEE Transactions on Power Electronics, vol. 29, no. 11, pp. 5795-5805, Nov. 2014.

[4] M. Hanif, V. Khadkikar, and W. Xiao, "Two degrees of freedom active damping technique for LCL filter-based grid connected PV systems," in IEEE Transactions on Industrial Electronics, vol. 61, no. 6, pp. 27952803, Jun. 2014.

[5] C. Bao, X. Ruan, and X. Wang, "Step-by-step controller design for LCLtype grid-connected inverter with capacitor-current-feedback activedamping," in IEEE Transactions on Power Electronics, vol. 29, no. 3, pp. 1239-1253, Mar. 2014.

[6] C. P. Dick, S. Richter, M. Rosekeit, M. Rolink, and R. W. De Doncker, "Active damping of LCL resonance with minimum sensor effort by means of a digital infinite impulse response filter," in 2007 European Conference on Power Electronics and Applications, 2007, pp. 1-8.

[7] X. Li, J. Fang, Y. Tang, X. Wu, and Y. Geng, "Capacitor-voltage feedforward with full delay compensation to improve weak grids adaptability of LCL-filtered grid-connected converters for distributed generation systems," in IEEE Transactions on Power Electronics, vol. 33, no. 1, pp. 749-764, Jan. 2017

[8] S. G. Parker, B. P. McGrath, and D. G. Holmes, "Regions of active damping control for LCL filters,"in IEEE Transactions on Industry Applications, vol. 50, no. 1, pp. 424 432, Jan.-Feb.2014.

[9] Y. Tang, P. C. Loh, P. Wang, F. H. Choo, F. Gao, and F. Blaabjerg, "Generalized design of high performance shunt active power filter with output LCL filter," in IEEE Transactions on Industrial Electronics, vol. 59, no. 3, pp. 1443-1452, Mar. 2012.

[10] W. Wu, J. Liu, Y. Li, and F. Blaabjerg,"Individual channel design-based precise analysis and design for three-phase grid-tied inverter with LCLfilter under unbalanced grid impedance," in IEEE Transactions on Power Electronics, vol. 35, no. 5, pp. 5381-5396, May 2020.

[11] J. Wang, J. D. Yan, L. Jiang, and J. Zou, "Delay-dependent stability of single-loop controlled grid-connected inverters with LCL filters," in IEEE Transactions on Power Electronics, vol. 50, no. 1, pp. 743-757, Jan. 2016.

[12] Y. Chen, Z. Xie, and L. Zhou, "Optimized design method for grid-currentfeedback active damping to improve dynamic characteristic of LCL-type grid-connected inverter," in International Journal of Electrical Power \& Energy Systems, vol. 100, pp. 19-28, 2018.

[13] X. Li, X. Wu, Y. Geng, X. Yuan, C. Xia, and X. Zhang, "Wide damping region for LCL-type grid-connected inverter with an improved capacitorcurrent-feedback method," in IEEE Transactions on Power Electronics, vol. 30, no.9, pp. 5247-5259, Sep. 2015.

[14] Y. Tang, P. C. Loh, P. Wang, F. H. Choo, and F. Gao, "Exploring inherent damping characteristic of LCL-filters for three-phase grid-connected voltage source inverters," in IEEE Transactions on Power Electronics, vol. 27, no. 3, pp. 1433-1443, Mar. 2012.

[15] S. Yang, Q. Lei, F. Z. Peng, and Z. Qian, "A robust control scheme for grid-connected voltage-source inverters," in IEEE Transactions on Industrial Electronics. vol. 58, no. 1, pp. 202-212, Jan. 2010.

[16] Z. Xin, X. Wang, P. C. Loh, and F. Blaabjerg, "Grid-current-feedback control for LCL-filtered grid converters with enhanced stability," in IEEE Transactions on Power Electronics, vol. 32, no. 4, pp. 3216-3228, Apr. 2017.

[17] D. M. VandeSype, K. DeGusseme, F. M. L. L. DeBelie, A. P. VandenBossche, and J. A. Melkebeek, "Small-signal z-domain analysis of digitally controlled converters," in IEEE Transactions on Power Electronics, vol. 21, no. 2, pp. 470-478, Mar. 2006.

[18] Z. Xin, P. Mattavelli, W. Yao, Y. Yang, F. Blaabjerg, and P. C. Loh, "Mitigation of grid-current distortion for LCL-filtered voltage-source inverter with inverter-current feedback control," in IEEE Transactions on Power Electronics, vol. 33, no. 7, pp. 6248-6261, Jul. 2018.

[19] R. A. Fantino, C. A. Busada, and J. A. Solsona, "Grid impedance estimation by measuring only the current injected to the grid by a VSI with LCL filter," in IEEE Transactions on Industrial Electronics, vol. 68, no. 3, pp. 1841-1850, Mar. 2021.

[20] D. Pan, X. Ruan, X. Wang, F. Blaabjerg, X. Wang, and Q. Zhou, "A highly robust single-loop current control scheme for grid-connected inverter with an improved LCCL filter configuration," in IEEE Transactions on Power Electronics, vol. 33, no. 10, pp. 8474-8487,
Oct.2018.

[21] R. A. Fantino, C. A. Busada, and J. A. Solsona, "Observer-based gridvoltage sensorless synchronization and control of a VSI-LCL tied to an unbalanced grid," in IEEE Transactions on Industrial Electronics, vol. 66 , no. 7, pp. 4972-4981, Jul. 2019.

[22] L. Zhou, X. Zhou, Y.Chen, Z. Lv, Z. He,W. Wu, L. Yang, K.Yan, A. Luo,andJ. M. Guerrerohttps://orcid.org/0000-0001-5236-4592, "Invertercurrent-feedback resonance-suppression method for LCL-type DG system to reduce resonance-frequency offset and grid-inductance effect," in IEEE Transactions on Industrial Electronics, vol.65, no. 9, pp. 70367048, Sep. 2018.

[23] Q. Wang, W. Qin, and X. Han, "Robustness enhanced with damping interval widening strategy of LCL-type converter under weak grid condition," in Journal of Power Electronics, vol. 20, no. 2, pp. 410-427, 2020

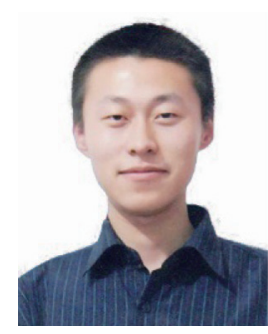

Qi Wang was born in Shanyin, China, in 1991. He received his B.S., M.S. and Ph.D. degrees in electrical engineering from the Taiyuan University of Technology, Taiyuan, China, in 2015, 2017, and 2020 respectively. He is presently working as an Assistant Professor at Shanxi University. His current research interests include the stability analysis of renewable energy grid-connected converters under complex grid conditions, power quality of microgrids, and power system operation and control. He is a Member of IEEE.

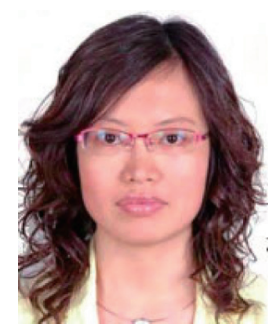

Wenping Qin was born in China, in 1972. She received her B.S., M.S. and Ph.D. degrees from the Taiyuan University of Technology (TYUT), Taiyuan, China, in 1995, 2001 and 2015, respectively. She is presently working as a Professor in TYUT. Her current research interests include power system reliability assessment, stability and security analysis, power system protection and microgrid technology. She is a Member of IEEE.

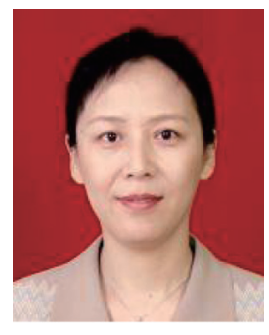

Xiaoqing Han received her B.S., M.S. and Ph.D. degrees from the College of Electrical and Power Engineering, Taiyuan University of Technology, Taiyuan, China. She is presently working as a Professor at the Taiyuan University of Technology. Her current research interests include power system simulations, stability analysis, and the integration of renewable energy sources. Professor Han was a recipient of Science and Technology Awards of Shanxi Province, China, in 2001 and 2005. She is a Member of IEEE.

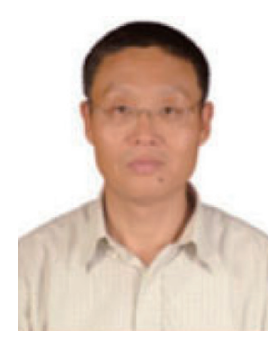

Peng Wang received his B.S. degree from Xi'an Jiaotong University, Xi'an, China, in 1978; his M.S. degree from the Taiyuan University of Technology, Taiyuan, China, in 1987; and his M.S. and Ph.D. degrees from the University of Saskatchewan, Saskatoon, SK, Canada, in 1995 and 1998, respectively. $\mathrm{He}$ is presently working as a Professor in the School of Electrical and Electronic Engineering, Nanyang Technology University, Nanyang, Singapore. He is a Fellow of IEEE. 


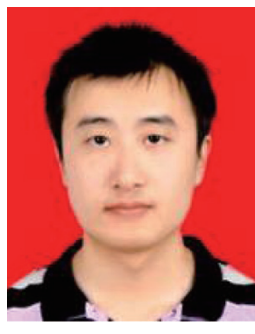

Lei Wang was born in China, in 1985. He received his M.S. and Ph.D. degrees from the Taiyuan Universityof Technology, Taiyuan, China, in 2010 and 2017, respectively. He is presently working as an Assistant Professor in the College of Electrical and Power Engineering, Taiyuan University of Technology. His current research interests include power electronic converters, active and hybrid filters, the application of power electronics in renewable energy systems, and power quality compensation systems.

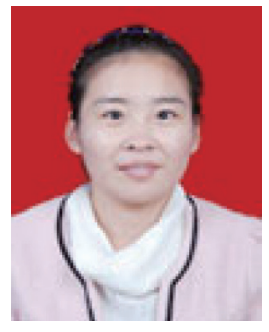

Ye Zhang was born in Hebei, China, in 1987. She received the B.S. degree in electrical engineering from China, University of Mining and Technology, China in 2011, the M.S. degree from Tianjin University, China, in 2014. Her main interest includes power quality analysis and control of power converters for renewable energy systems. 